\title{
The invocation of the Archangels Michael and Gabriel attributed to Metrophanes metropolitan of Smyrna (BHG 1292)
}

Abstract: This article presents a critical edition and annotated English translation of the Invocation of the holy Archangels Michael and Gabriel by Metrophanes, who was bishop of Smyrna in the second half of the 9th century. The text has only been preserved in the 12th-century manuscript Oxoniensis, Bodleianus, Auctarium E.5.12 (Miscellaneus 77). This new reconstruction of the Greek text replaces the unreliable edition of 1887 by Basileios Georgiadès. In the notes accompanying the translation, references to expressions and Biblical quotes recurrent in the oeuvre of Metrophanes have been added.

Adresse: Dr. Erika Gielen, Blijde Inkomststraat 5 (bus 3004), 3000 Leuven, BELGIUM; erika.gielen@hiw.kuleuven.be - Prof. Dr. Peter Van Deun, Blijde Inkomststraat 21 (bus 3318), 3000 Leuven, BELGIUM; peter.vandeun@arts.kuleuven.be.

The Byzantine author Metrophanes is no stranger to us. In two previous articles, we uncovered formerly unknown texts of this bishop of Smyrna. ${ }^{1}$ About his life, however, little is known. As one of Photius' chief opponents, he lost his see in Smyrna after the Photian Synod in 859. Having suffered imprisonment and forced exile, he was allowed to return to his see in Smyrna several years later, in 867. In this way, he was one of the key figures of the anti-Photian Council of 869-870. However, about nine years later, he was exiled once more just before the Photian Synod of 879-880. Nothing is known about the last years of his life, except for the fact that his date of death must have been before October 912 .

Until our publications, his literary œuvre amounted to just a few pages: some liturgical texts; a Letter to the logothete Manuel on the deposition of Pho-

1 P. VAn Deun, La chasse aux trésors: la découverte de plusieurs œuvres inconnues de Métrophane de Smyrne (IX ${ }^{\mathrm{e}}-\mathrm{X}^{\mathrm{e}}$ siècle). Byz 78 (2008) 346-367. See also P. VAN Deun, Trésors inconnus de la littérature byzantine des $\mathrm{IX}^{\mathrm{e}}-\mathrm{X}^{\mathrm{e}}$ siècles. Annuaire EPHE. Sciences Religieuses 117 (2008-2009) 273-276; Metrophanes (25088) in R.-J. LiLIE et al., Prosopographie der mittelbyzantinischen Zeit. Zweite Abteilung (867-1025), 4. Band. Berlin 2013, 437-439. 
tius; the Panegyric on Polycarp of Smyrna; ${ }^{2}$ and the Laudatio archangelorum Michael et Gabriel, a critical edition of which can be found in this article. In our 2008 article, we proved that several other texts, most of which are very long,

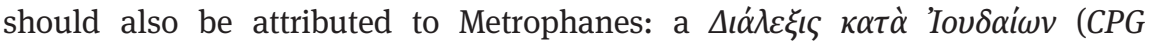
7799), anonymously preserved in the codex unicus Florentinus, Mediceus-Laurentianus plut. 7, 1 (first half of the 10th century) and dated to the years 907-908 (the reign of Emperor Leo VI); ${ }^{3}$ a very long Commentary on Ecclesiastes (CPG 7950), which has come down to us in four Greek manuscripts ${ }^{4}$; Homilies on the Gospels of St John and St Matthew, the edition of which could be made thanks to one sole manuscript, the Londinensis, British Library, Additional 39605 (beginning of the 10th century) 5 and dated between February 907 (the deposition of Patriarch Nicholas Mystikos) and May 912 (the death of emperor Leo VI); ${ }^{6}$ the fragmentary

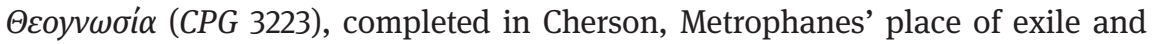
mainly preserved in the 12th-century Panoplia dogmatica of Euthymios Zygabènos; ${ }^{7}$ a Commentary on the Catholic Letters, largely unedited and preserved once again in only one manuscript, viz Athous Dionysiou 227 (15th century); an unedited Commentary on Hexaemeron; a Psalm Commentary, fragments of which have been preserved in the catenatic literature on Psalms; the Professio fidei, allegedly by Metrophanes' hand, which has come down to us in one manuscript and the first edition of which is currently being prepared in Leuven. ${ }^{8}$

2 I. De Vos / P. Van Deun, The panegyric of Polycarp of Smyrna attributed to Metrophanes of Smyrna (BHG 1563) in J. Leemans (ed.), Martyrdom and persecution in late antique Christianity. Festschrift Boudewijn Dehandschutter. Bibliotheca Ephemeridum Theologicarum Lovaniensium, 241. Leuven/Paris/Walpole, MA 2010, 311-331.

3 Edited by M. Hostens, Anonymi auctoris Theognosiae (saec. IX/X) Dissertatio contra Iudaeos. CCSG, 14. Turnhout/Leuven 1986.

4 Edited by G.H. EtTlinger / J. Noret, Pseudo-Gregorii seu Pseudo-Gregorii Nysseni Commentarius in Ecclesiasten. CCSG, 56. Turnhout/Leuven 2007. We discovered a Georgian translation of this work, unknown to the editors of the Greek text; for an edition, see K. KeKeLIDZE, Commentarii in Ecclesiasten Metrophanis, Metropolitae Smyrnensis, Monumenta Georgica. Publicationes Universitatis Tphilisensis, 1. Scriptores Ecclesiastici, 1. Tiflis 1920. There the Commentary is attributed to Metrophanes of Smyrna.

5 In this manuscript, the name of the author has been completely effaced.

6 Edited by K. HANSmanN, Ein neuentdeckter Kommentar zum Johannesevangelium. Untersuchungen und Text. Forschungen zur Christlichen Literatur- und Dogmengeschichte, 16, 4/5. Paderborn 1930.

7 In the Panoplia, the fragments are attributed to Gregory of Nyssa. P. Van Deun is currently pre-

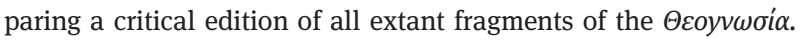

8 S. NeIRynck / P. Van Deun, Est-ce qu'on a découvert la profession de foi de Métrophane de Smyrne?, forthcoming by Brepols Publishers. 
Metrophanes suffered from a kind of damnatio memoriae, and so one can easily understand that most of his works have been transmitted only very poorly - very often, there is just a single manuscript of a certain text. Recent research, however, proves that he was by no means a man of no importance in the theological and cultural life of the late 9th and early 10th century. It even might be no exaggeration to state that he was his most famous contemporary's, Photius, equal.

The Invocation of the Archangels Michael and Gabriel (BHG 1292) is not completely unknown, since it was published in 1887 by Basileios Georgiadès, eventually Patriarch Basil III. ${ }^{9}$ Unfortunately his edition is unreliable. The text has only been preserved in the parchment codex Oxoniensis, Bodleianus, Auctarium E.5.12 (Miscellaneus 77), a miscellany of some ascetical (e.g. the Pandects of Antiochus the Monk and Doctrina XV of Dorotheus of Gaza) and 17 hagiographical texts, written by different scribes, probably at the end of the 12 th century. ${ }^{10}$ The text of Metrophanes is to be found on f. $250-256^{\mathrm{v}}$.

Since Metrophanes is mentioned as the author in the manuscript itself, there is no doubt about the authenticity of the text. This claim is confirmed by the actual language of the text, as it is characterized by various unusual phrasings that are, however, recurrent in Metrophanes' oeuvre. Moreover, several Bible verses that are quoted in this text also occur in other treatises of Metrophanes, and there are also some overlaps as regards content. All these cases are listed below in the footnotes explaining the English translation accompanying the critical edition of the text. ${ }^{11}$

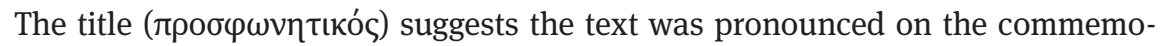
ration day of the Archangels Michael and Gabriel celebrated on November 8th. There is, however, no information about the place where it was delivered (Smyrna, Constantinople?).

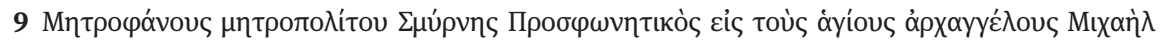

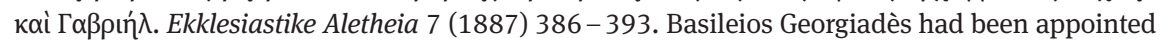
editor in chief of this journal previously, in 1884.

10 The best description of the manuscript was made by H.O. CoxE, Catalogi codicum manuscriptorum Bibliothecae Bodleianae, 1, recensionem codicum graecorum continens. Oxford 1853 (reprint, with corrections, Oxford 1969), 659-661; see also C. VAN DE VoRST / H. DeLEHAYE, Catalogus codicum hagiographicorum graecorum Germaniae, Belgii, Angliae. Subsidia hagiographica, 13. Brussels 1913, 364-365.

11 The translation is ours, except for the quotations of the Septuagint. For these we used the New English Translation of the Septuagint (NETS). In the apparatus criticus, purely phonetic errors have not been included, unless the misspelled form is an actually existing word with a different meaning or a different grammatical value. 
The absence of any concrete details about Michael and Gabriel or about their miracle working is typical of our text. We did not find any evidence that Metrophanes might have used one of the existing Michael and Gabriel texts. Therefore this work does not occupy an important place in the hagiographic "dossier" on the Archangels. Nevertheless, the text testifies to the popularity of Michael (and Gabriel) in the 9th century ${ }^{12}$ and displays several well-known epitheta for the Archangels Michael and Gabriel, a large number of which pertain to the military field (e.g.

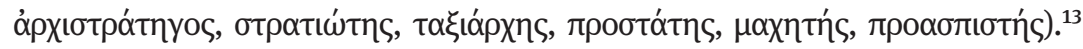

12 See, e.g., the article of B. MARTIN-Hisard, Le culte de l'archange Michel dans l'empire byzantin (VIII ${ }^{\mathrm{e}} \mathrm{XI}^{\mathrm{e}}$ siècles) in: Culto e insediamenti Micaelici nell'Italia Meridionale fra Tarda Antichità e Medioevo. Atti del Convegno Internazionale Monte Sant'Angelo 18-21 novembre 1992. Scavi e ricerche, 7. Bari 1994, 351-373.

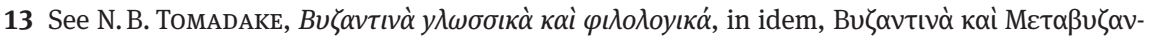

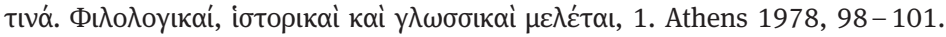




\section{Text}

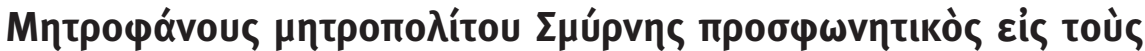

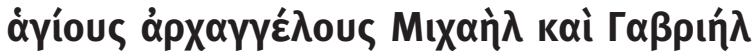

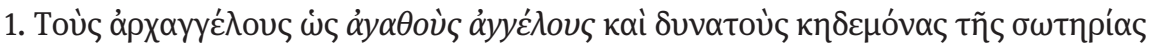

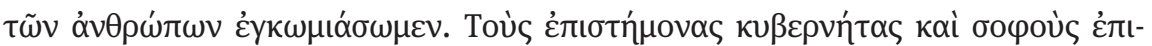

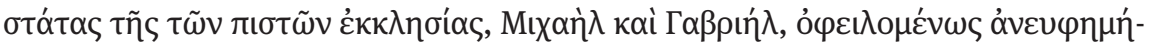

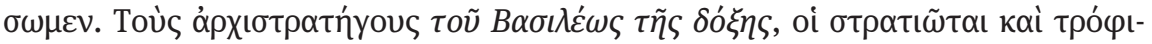

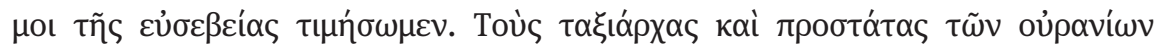

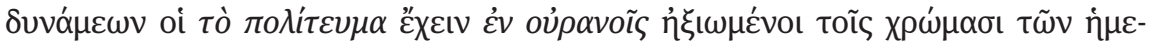

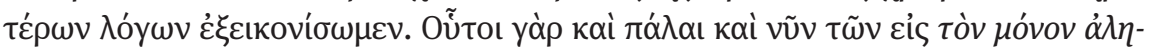

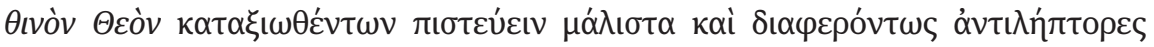

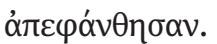

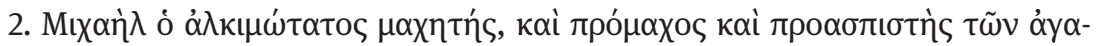

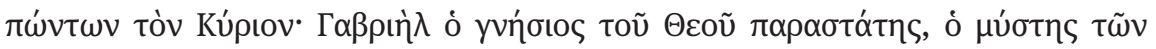

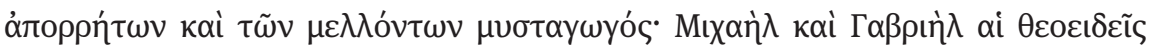

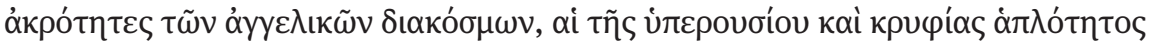

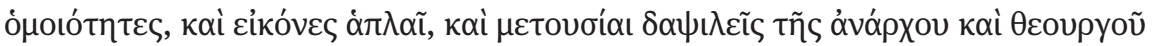

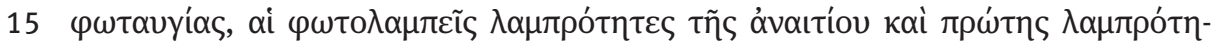

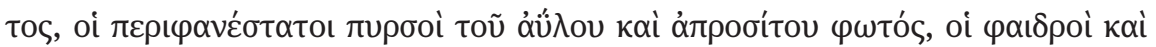

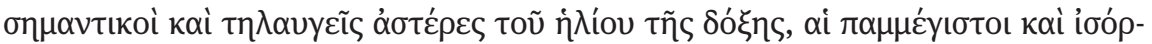

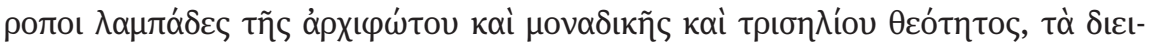

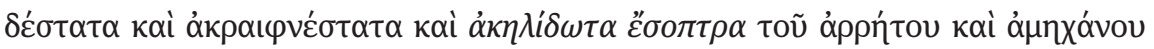

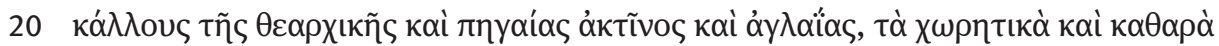

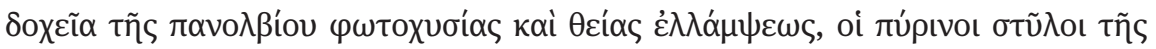

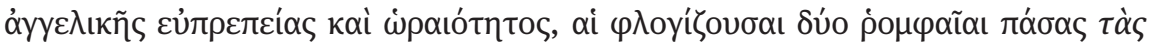

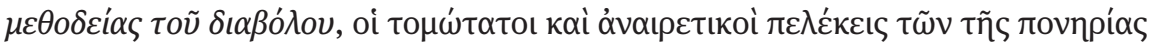

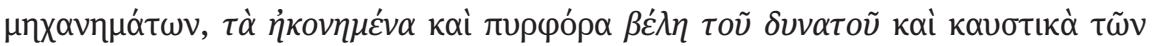

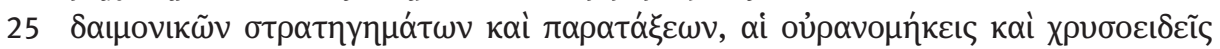

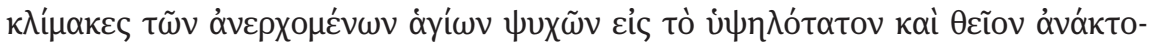

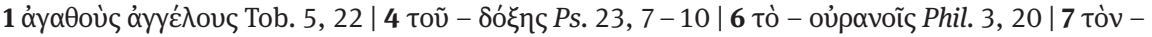

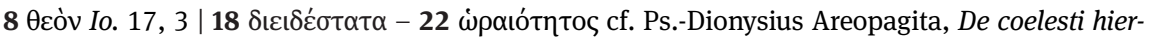
archia III, 2 (Corpus Dionysiacum, 2, eds. G. HeIL / A. M. RitTER. PTS, 36. Berlin / New York

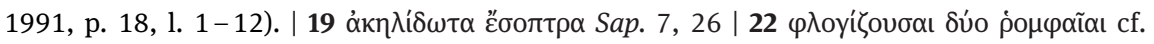

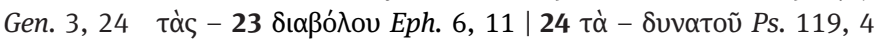

23 ávaıpetoì cod. et Georgiadès 


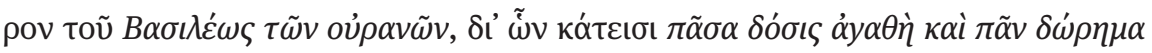

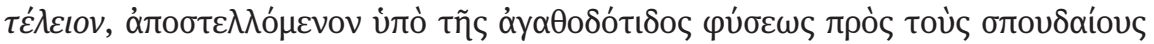

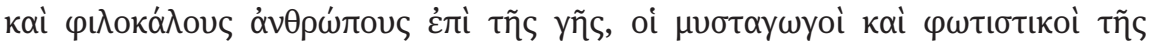

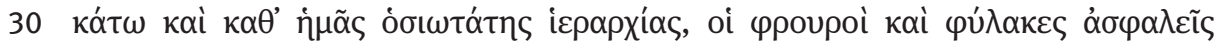

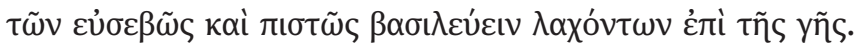

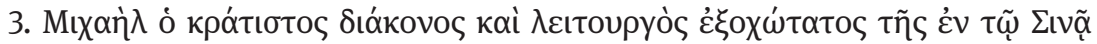

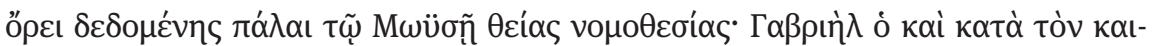

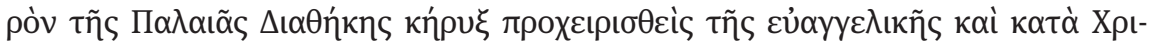

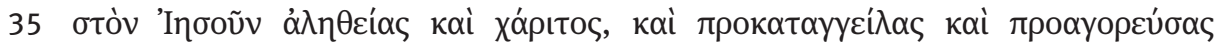

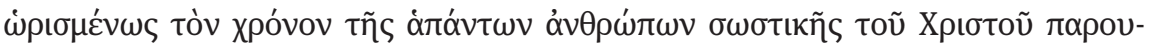

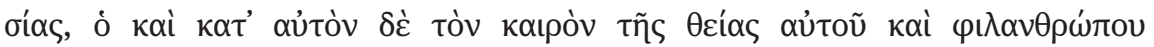

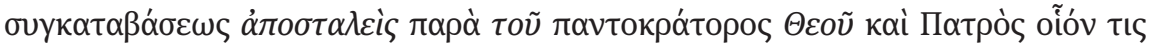

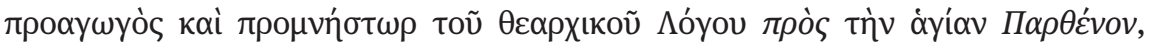

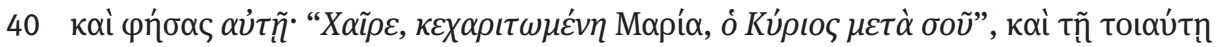

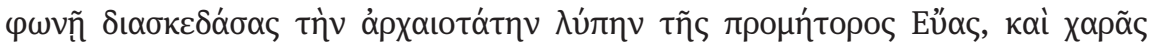

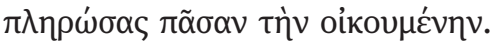

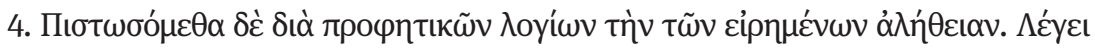

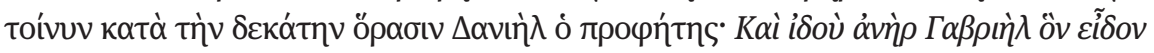

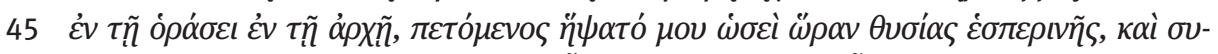

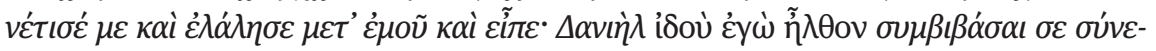

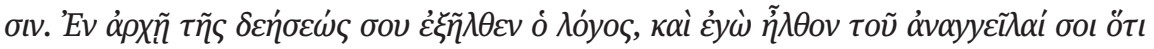

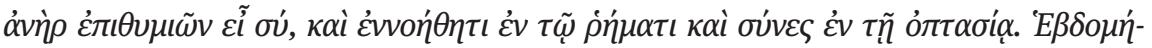

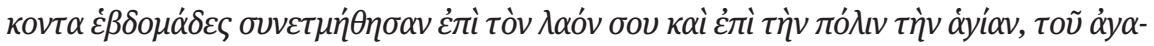

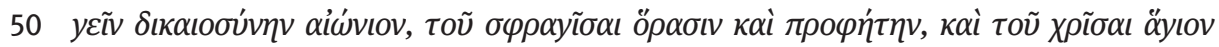

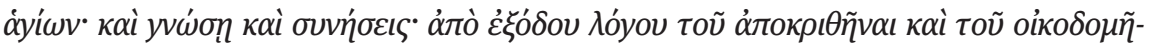

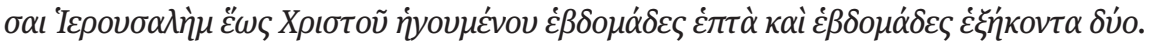

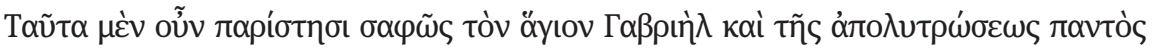

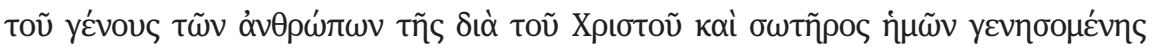

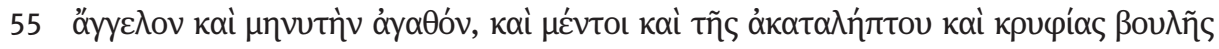

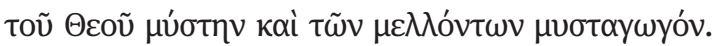

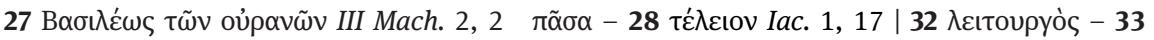

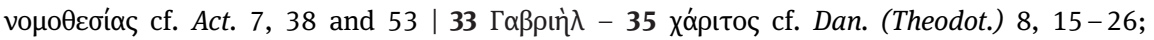

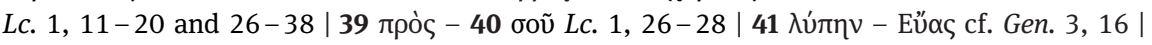
44 Kaì - 52 dúo Dan. (Theodot.) 9, 21-25

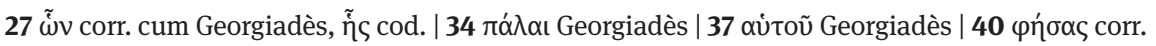

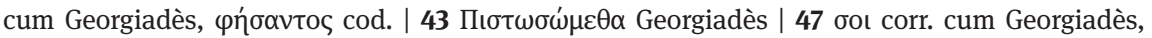
oov cod. 


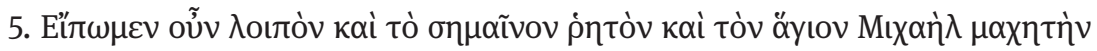

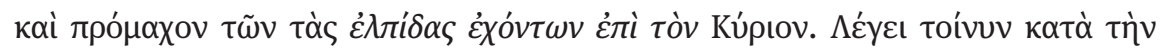

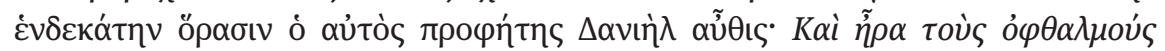

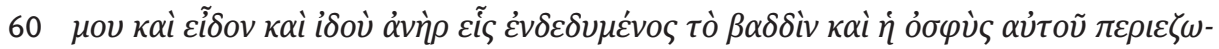

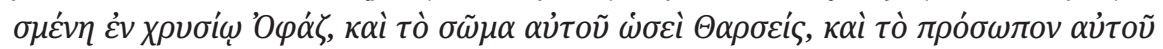

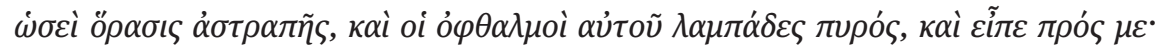

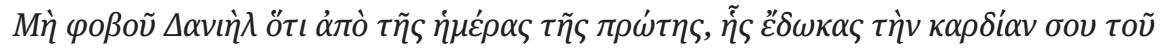

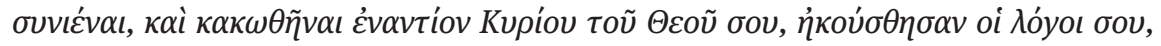

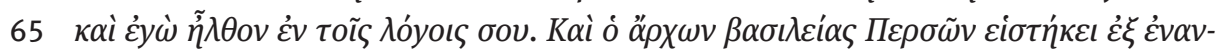

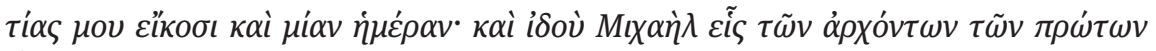

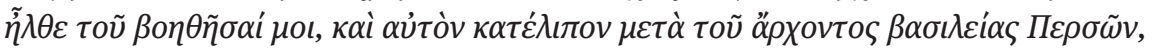

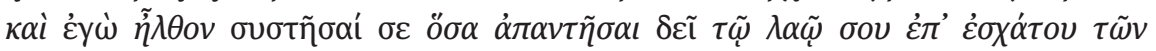

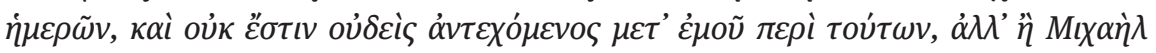

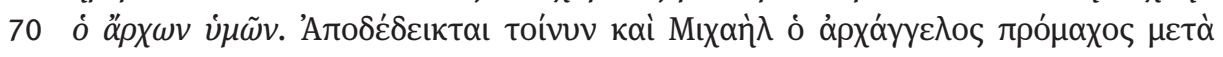

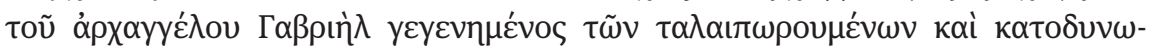

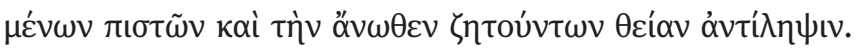

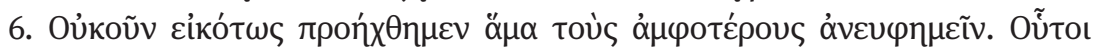

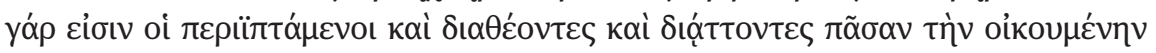

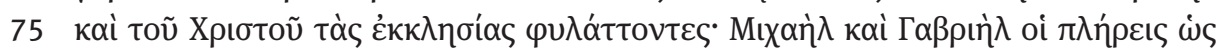

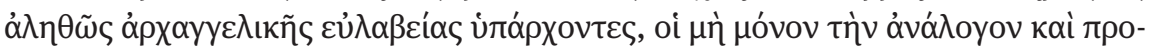

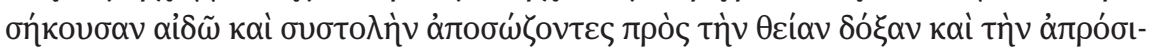

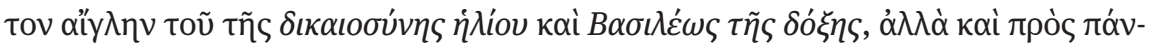

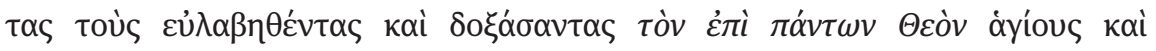

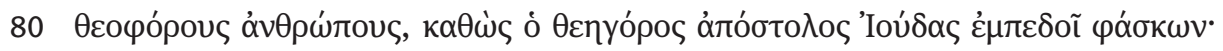

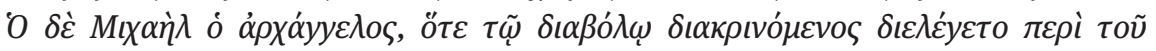

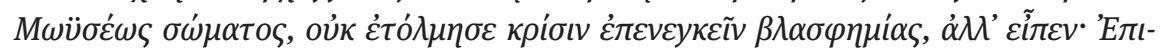

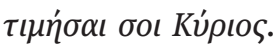

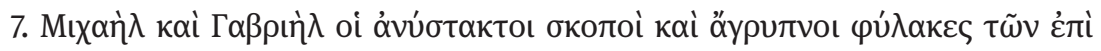

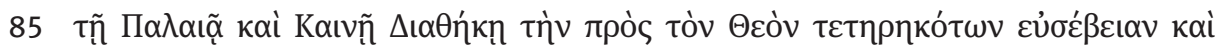

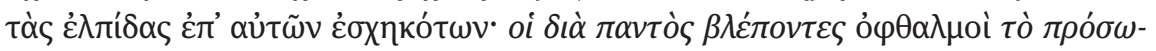

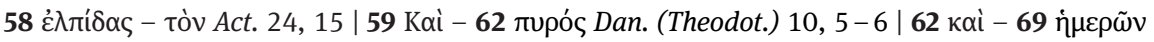

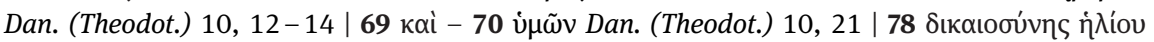

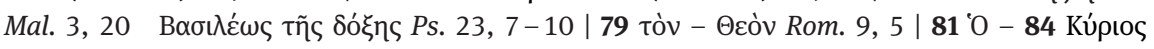
Ep. Iud. 9 | $\mathbf{8 6}$ oi $-\mathbf{8 8}$ oủpavoĩs Mt. 18, 10

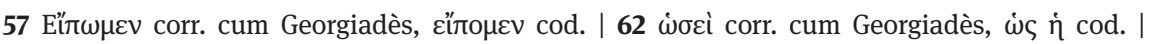

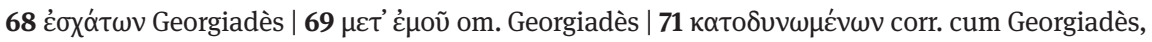

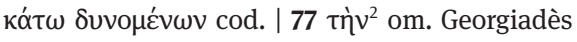




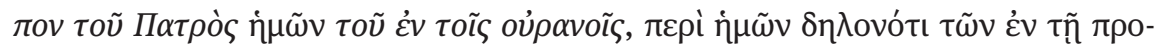

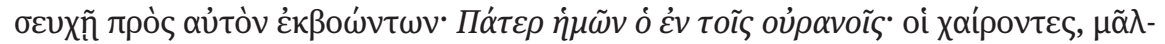

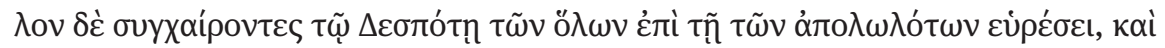

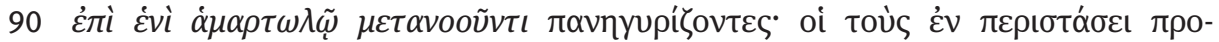

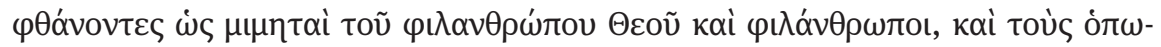

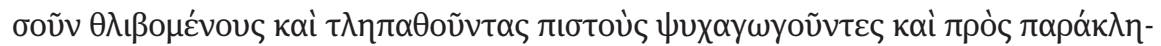

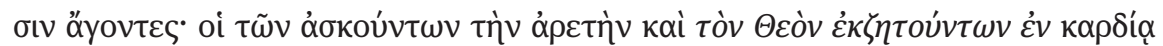

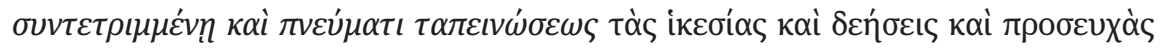

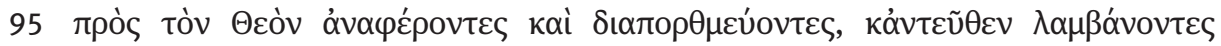

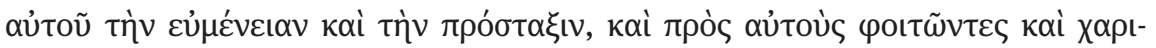

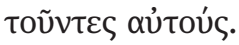

8. Мıх

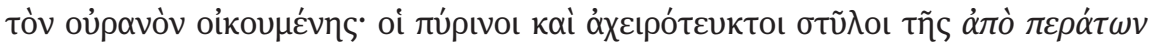

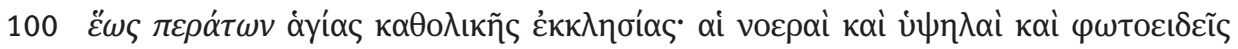

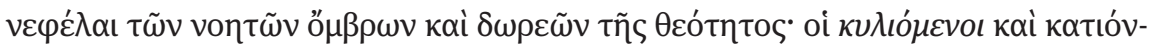

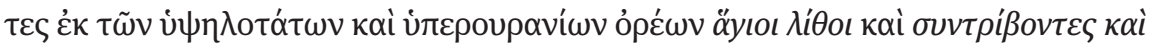

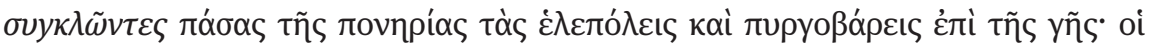

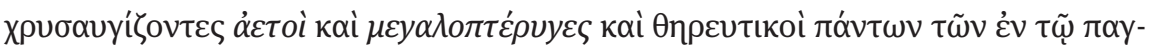

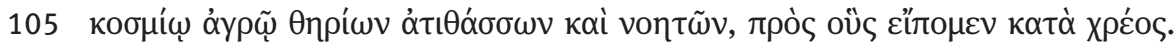

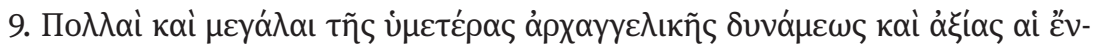

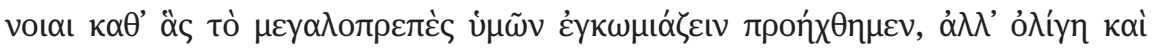

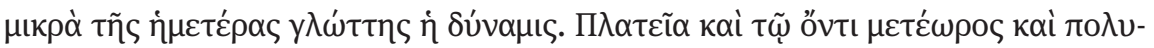

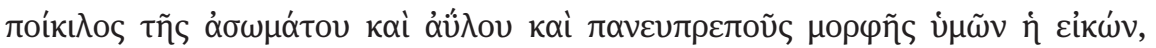

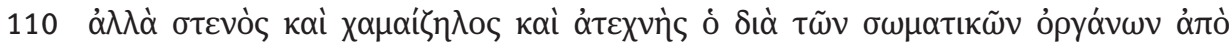

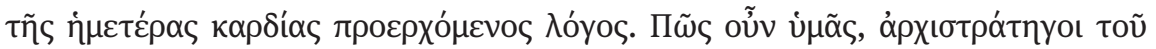

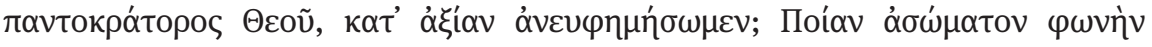

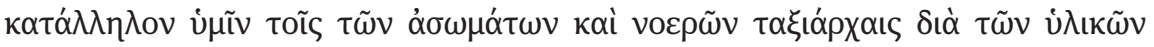

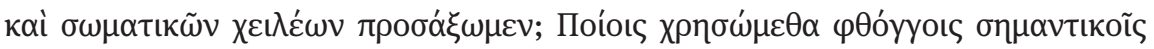

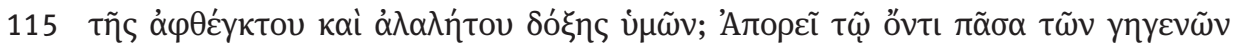

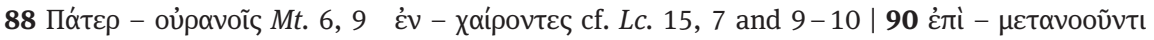

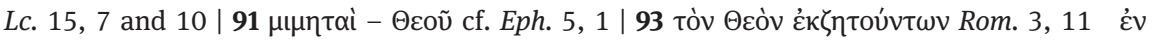

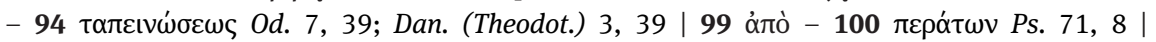

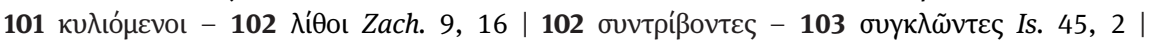

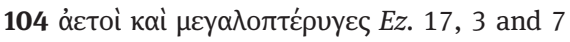

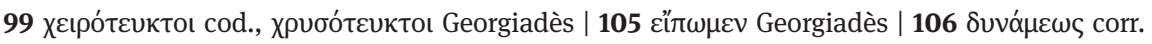

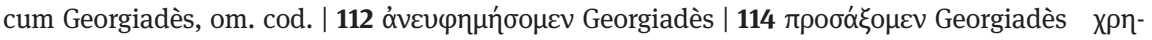
бó $\mu \varepsilon \theta \alpha$ Georgiadès 


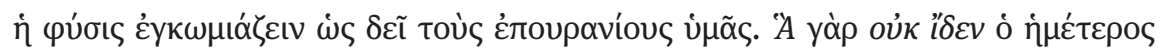

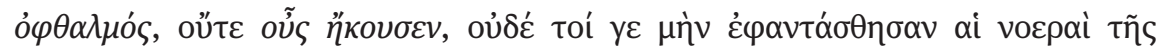

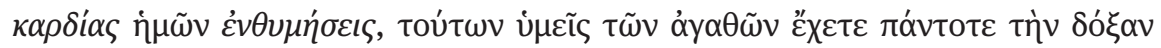

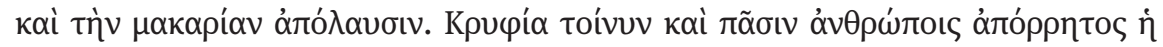

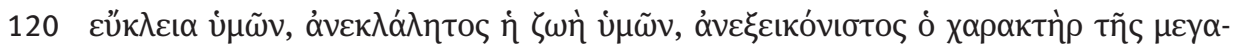

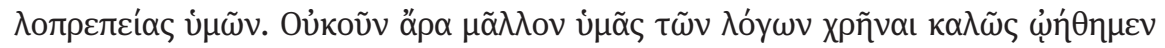

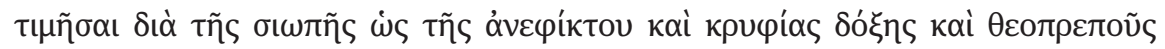

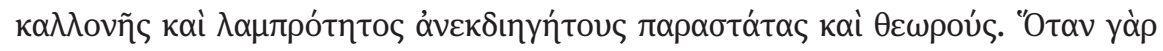

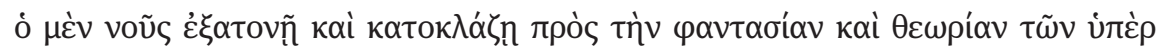

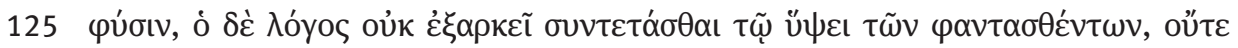

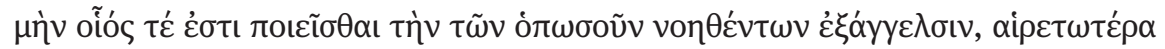

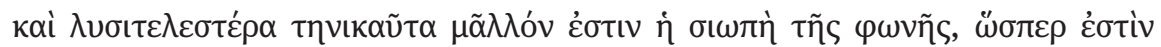

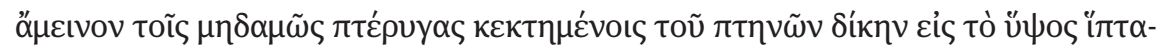

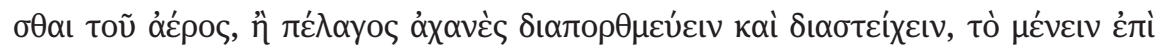

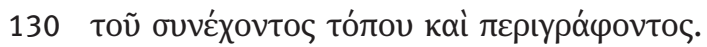

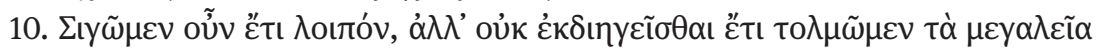

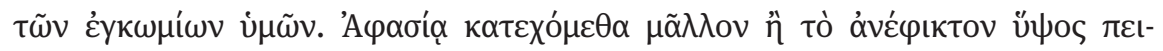

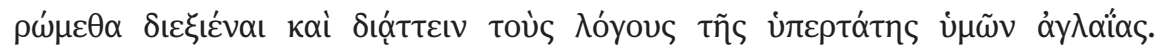

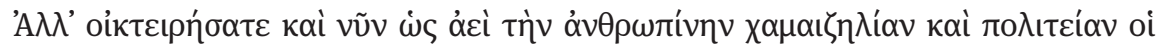

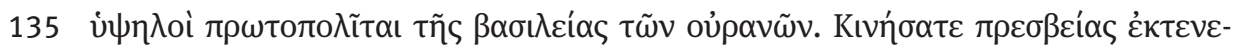

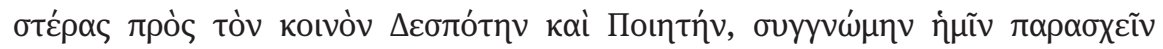

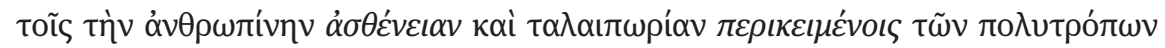

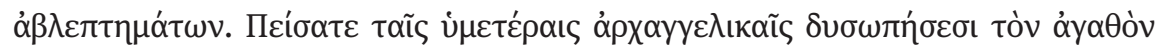

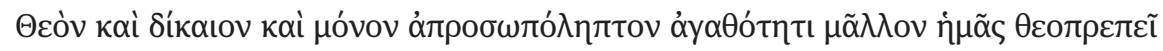

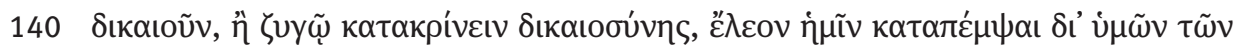

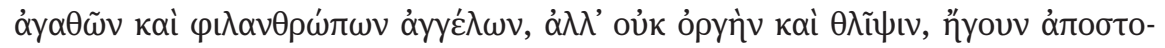

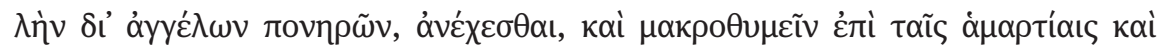

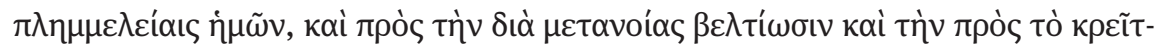

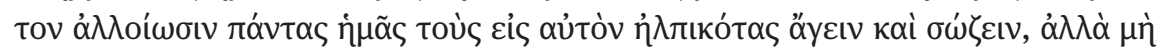

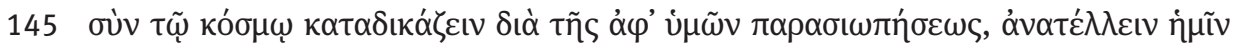

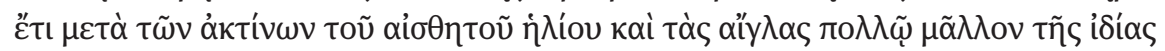

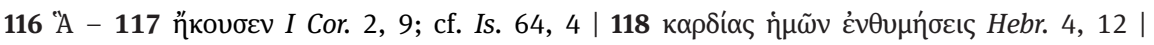

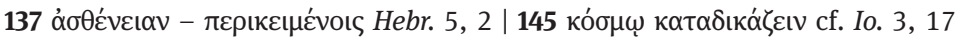

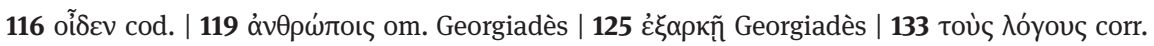

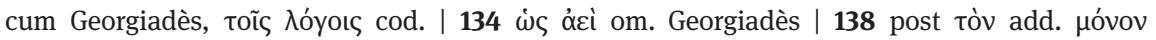
Georgiadès | 145 ن் $\mu \tilde{\omega} v$ corr. cum Georgiadès, ì $\mu \tilde{\omega} v$ cod. 


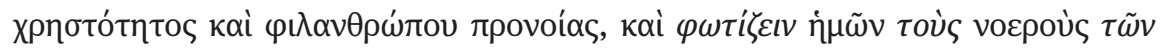

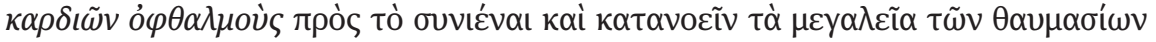

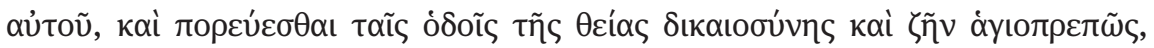

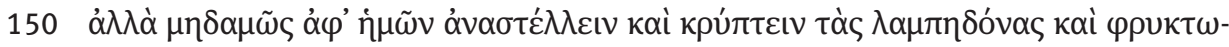

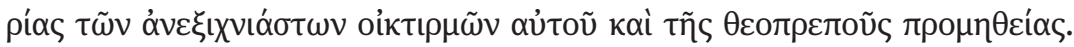

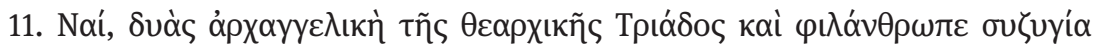

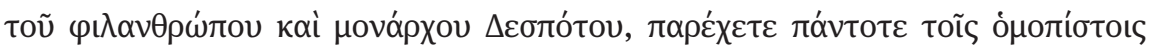

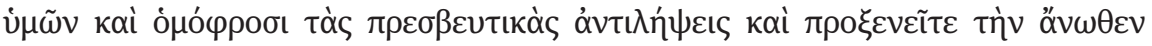

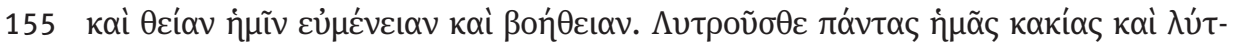

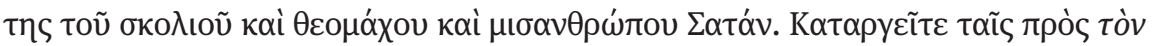

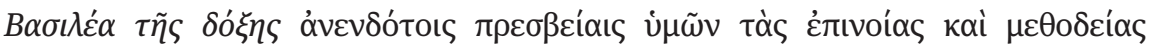

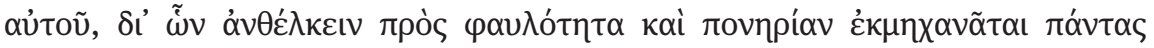

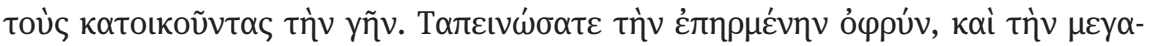

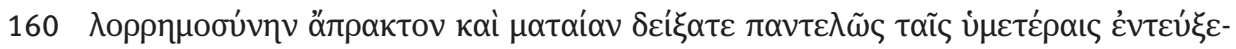

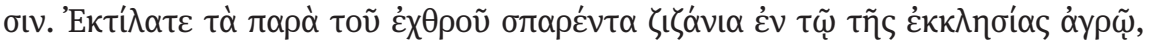

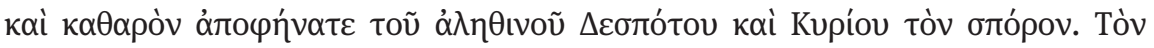

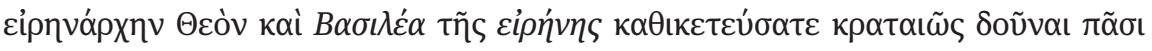

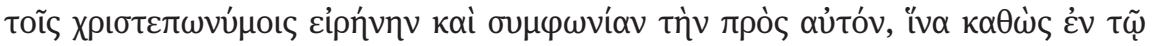

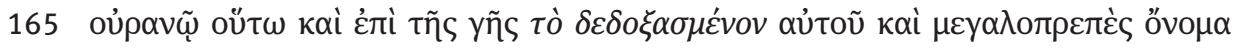

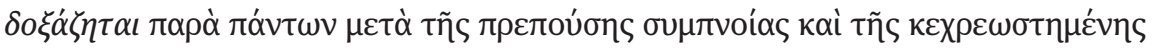

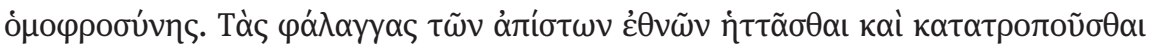

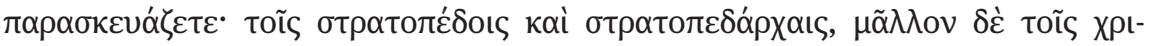

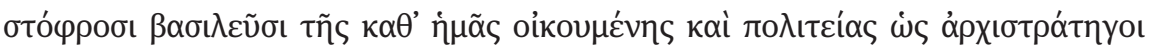

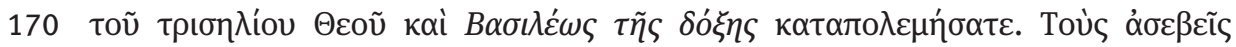

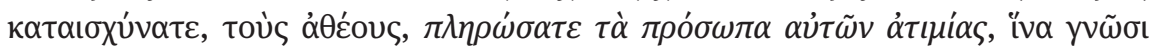

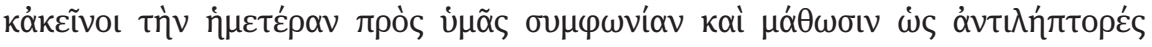

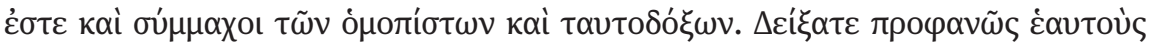

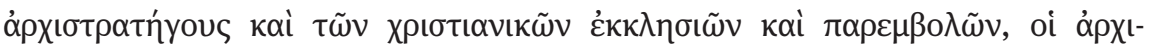

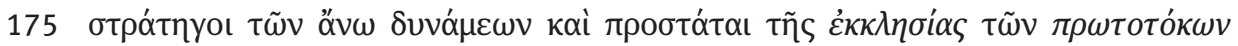

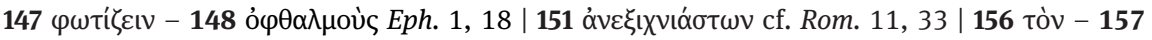

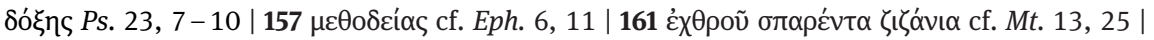

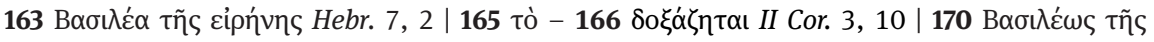

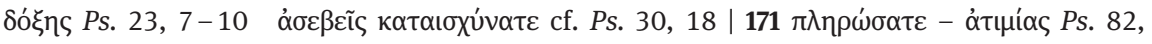

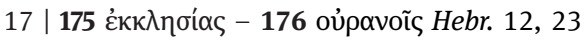

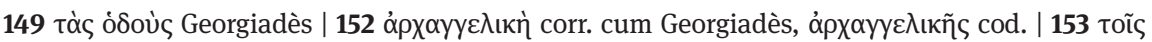

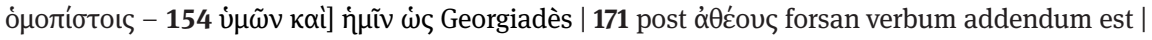

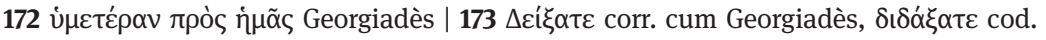




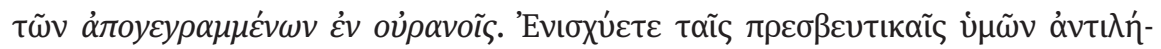

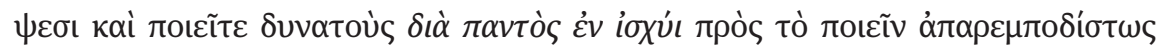

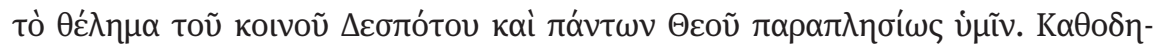

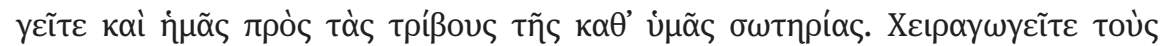

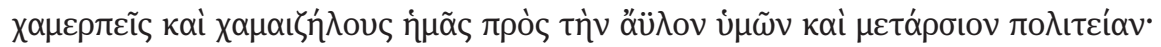

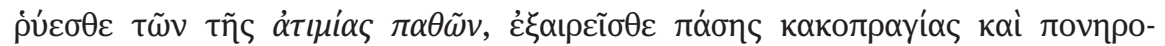

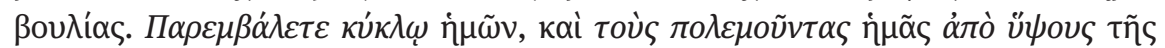

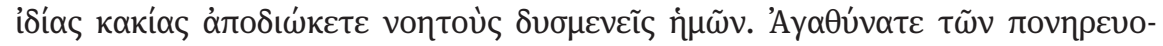

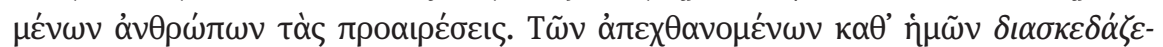

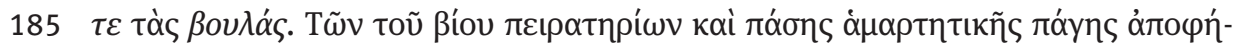

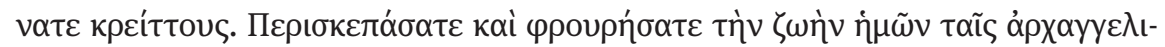

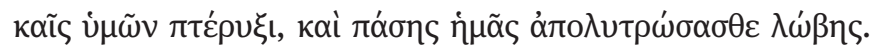

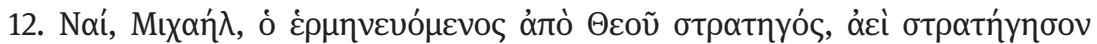

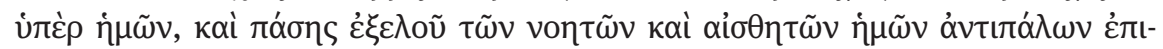

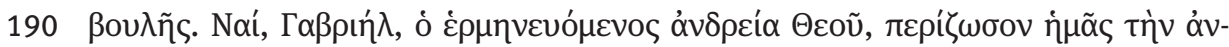

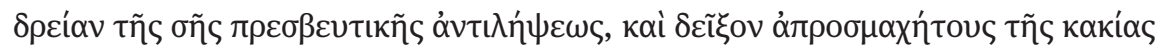

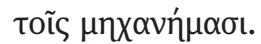

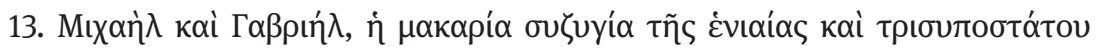

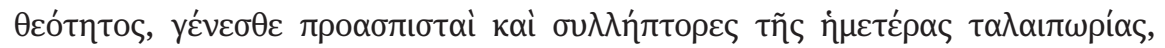

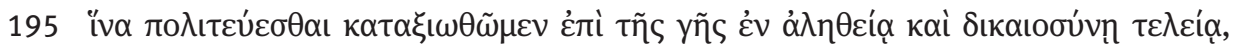

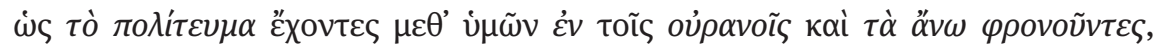

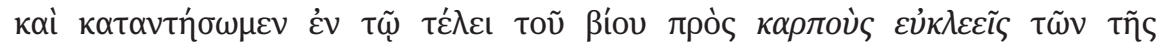

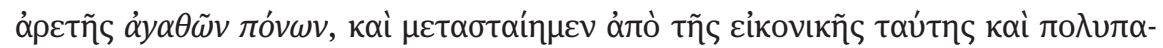

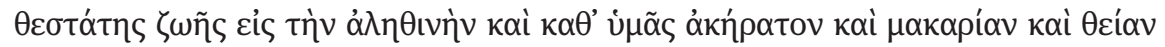

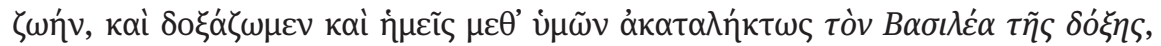

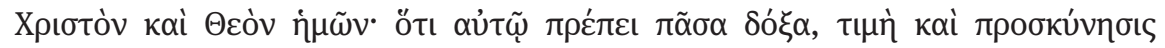

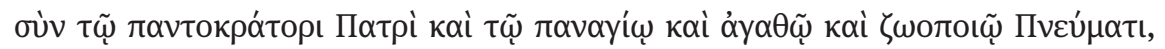

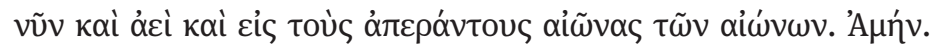

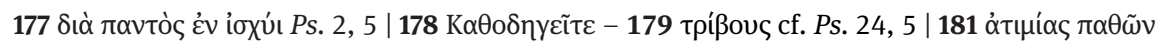

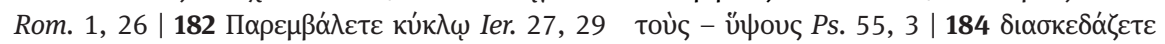

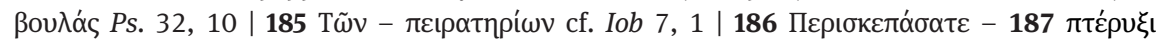

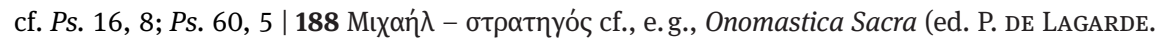

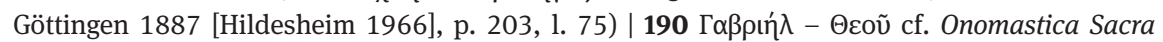

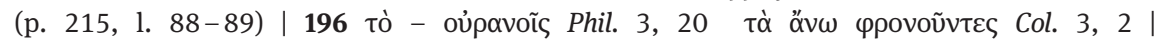

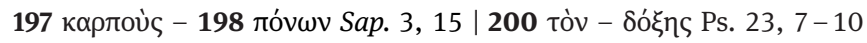

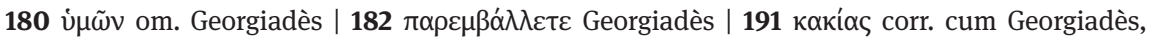

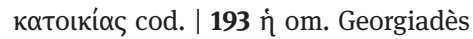




\section{Translation}

\section{Invocation of the holy Archangels Michael and Gabriel of Metrophanes, metropolitan of Smyrna}

1. Let us praise the Archangels as good messengers and mighty guardians of men's salvation. Let us duly laud the prudent guides and wise presidents of the church of the faithful, Michael and Gabriel. Let us, soldiers and nurslings of the true faith, ${ }^{1}$ honor the leaders of the King of glory. ${ }^{2}$ Let us, deemed worthy to hold citizenship in heaven, ${ }^{3}$ portray the commanders and champions of the heavenly powers with the embellishments of our words. For in times past as well as now, they have been declared above all and pre-eminently ${ }^{4}$ helpers of those who were held worthy of believing in the one true God:

2. Michael, the bravest warrior, defender and champion of those loving the Lord; Gabriel, God's genuine right-hand man, initiated into the unspeakable and initiator into what is to come. ${ }^{5}$ Michael and Gabriel, the godlike summits of the angelic orders; the supra-essential and hidden simplicity's likes and simple images; plentiful participation in the eternal and divine resplendent light; shining brightness of the uncaused, first brilliance; the most splendid fires of the immaterial and inapproachable light; the bright, denoting and far-shining rays of the sun of glory; the greatest and equally balanced flames of the divinity that is the source of light, one single unity and of three suns; the clearest, purest and spotless mirrors of the unspeakable and inconceivable beauty of the divine and primal brightness and splendor; the suitable and clear receptacles of the blessed shedding of light and the divine illumination; the fiery pillars of the angelic majesty and beauty; the two swords burning all the devil's wiles; the sharpest axes that destroy the contrivances of evil; the sharp and fire-bearing arrows of the powerful one, which can burn down the demonic stratagems and battle lines;

1 A similar expression can be found in Metrophanes' In Ecclesiasten 6, $20,34$.

2 Metrophanes quotes this passage 7 times in his Adversus Iudaeos, 3 times in his Homiliae and

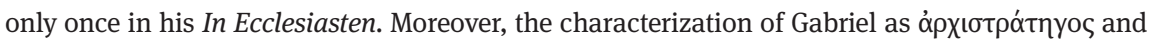

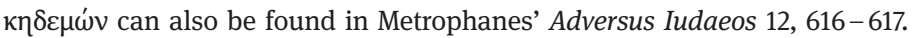

3 Passage quoted twice by Metrophanes in his Adversus Iudaeos and 4 times in his In Ecclesiasten.

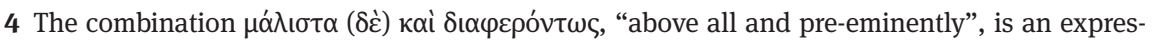
sion very typical of Metrophanes. See M. Hostens, Dissertatio (as footnote 3 above) xxviii; P. VAN Deun, La chasse (as footnote 1 above) 346-367, here 357.

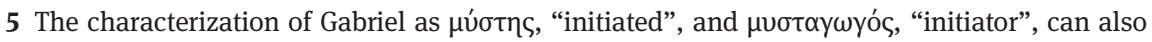
be found in Metrophanes' Adversus Iudaeos 12, 613-620 and 626-627. 
the sky-high, golden ladders of the holy souls ascending to the loftiest and divine house of the King of the heavens, from which all generous act of giving and every perfect gift comes down, ${ }^{6}$ sent down by good-giving nature to excellent and beauty loving men on earth; the initiators illuminating the most holy hierarchy from above and among us; the steadfast guards and sentinels of those chosen to rule devoutly and faithfully on earth. ${ }^{7}$

3. Michael, the strongest ministrant and mightiest servant of the divine legislation, in olden times given to Moses on Mount Sinai; Gabriel, already in those days of the Old Covenant appointed herald of the evangelic truth and grace according to Jesus Christ, as he foretold and prophesied definitely the time of Christ's coming, preserving all mankind, and who at the time of His divine descent as well, out of love for mankind, was sent from the almighty God and Father, as go-between and matchmaker ${ }^{8}$ of the divine Word and the blessed Virgin, saying to her: "Hail, favored Maria, the Lord is with you", and who, with such saying, brought to naught the original grief of first mother Eve, ${ }^{9}$ and filled the whole world with you.

4. We will confirm the truth of what has been said through sayings of the prophets. In his tenth vision, the prophet Daniel speaks as follows: “And lo, a man, Gabriel, whom I had seen in the vision in the beginning, flying, touched me about the time of the evening sacrifice, and he instructed me and spoke with me and said: 'Behold, Daniel, I have come to teach you understanding. At the beginning of your supplication, a word went out, and I have come to declare it to you, for you are a man of desires. And consider by means of the word and understand by means of the vision. 70 weeks have been cut short for your people and for the holy city to bring everlasting righteousness, to seal vision and prophet and to anoint a holy of holies. And you shall know and understand: from the going forth of the word to respond to and to rebuild Jerusalem until an anointed leader, there will be seven weeks and 62 weeks." "10 So, these words clearly present the holy ${ }^{11}$ Gabriel as messenger and good bringer of the news

6 This Bible verse is also quoted by Metrophanes in In Ecclesiasten 1, 22, 48.

7 This expression is also found in Metrophanes' In Ecclesiasten 9, 18, 10.

8 This combination of words can also be found in Metrophanes' Panegyricus Polycarpi p. 323, 1. 23.

9 The same thought can be found almost literally in the Oratio in nativitatem sanctae Dei genitricis Mariae (CPG 8060), attributed wrongly, so it seems, to John Damascene (ed. B. KoTTER, Die Schriften des Johannes von Damaskos, 5. PTS, 29. Berlin/New York 1988, 169, sect. 1, 1. 8-9). 10 The same passage, albeit with some differences, is quoted by Metrophanes in Adversus Iudaeos 12, 593-607.

11 From here until the end of the text, Georgiadès suggests to change öyıv ("holy") into öyyعhov ("messenger, angel"). According to us, however, such a change is not necessary. 
of the redemption of whole the human race, which will come about through Christ, our Savior, and, of course, as initiated in God's incomprehensible, hidden counsel, and as initiator into what is to come. ${ }^{12}$

5. Let us, then, further quote as well the passage which shows also the holy Michael as warrior and defender of those having hopes in the Lord. Thus the very same prophet Daniel speaks again in his eleventh vision: "And I raised my eyes, and saw, and lo, there was one man, clothed in baddin and his waist girded by gold of Ophaz; and his body was like Tharsis, and his face like an appearance of lightning, and his eyes like torches of fire. And he said to me: 'Do not fear, Daniel, for from the first day that you gave your heart to understanding and to be afflicted before your Lord God, your words have been heard, and I have come because of your words. And the ruler of the kingdom of the Persians stood opposite me 21 days. And lo, Michael, one of the chief rulers, came to help me, and I left him there with the ruler of the kingdom of the Persians, and I have come to instruct you about what will meet your people at the end of days. And there is not one with me who holds out against these things except Michael, your ruler." 'So, also the Archangel Michael has been proclaimed, together with the Archangel Gabriel, champion of the distressed and grievously afflicted faithful, looking for divine aid from above.

6. Surely, we were suitably induced to praise them both together. For it are these who fly, run and rush across the wole world, and guard Christ's churches, Michael and Gabriel, who, really filled with Archangels' discretion, maintain the proportionate and appropriate awe and humble position not only towards the divine glory and inapproachable splendor of the sun of justice and the King of glory, but also towards all the saints and God bearing men, who reverence and glorify the God of all, just as the apostle Judas, who speaks of God, confirms, ${ }^{13}$ saying: "But even when the Archangel Michael contended with the devil and disputed about the body of Moses, he did not dare to bring a condemnation of slander, but said: 'May the Lord rebuke you."'

7. Michael and Gabriel, the vigilant scouts and wakeful guards of those people who have watched over the reverence towards God in the Old and New Covenant and who have put their hopes on them. Their eyes always see the face of our Father in heaven, for us, of course, who in prayer call out to him: "Our Father, who is in heaven"; they rejoice, or rather, rejoice together with the Lord of everything at finding those who were lost, and take great pleasure in one sinner

12 Cf. supra, footnote 5.

13 Metrophanes likes to use the rare verbs $\varepsilon \mu \pi \varepsilon \delta \delta^{\prime} \omega$ and $\pi \alpha \rho \varepsilon \mu \pi \varepsilon \delta$ ó $\omega$, "to confirm, to corroborate”. See EtTlinger/ Noret, Commentarius (as footnote 4 above) li; VAn Deun, Métrophane (as footnote 1 above) 356 . 
who repents; they anticipate those who are in a difficult position as imitators of God who loves mankind, ${ }^{14}$ and by being human themselves; they lead the faithful who are somehow or other distressed and full of misery, and bring them consolation; they uplift and transmit the supplications, entreaties and prayers to God of those who act virtuously and seek God in their broken hearts and spirit of humiliation, and bring from there his favor and command, and resort to them and shed grace upon them.

8. Michael and Gabriel, the great and never-setting lights of the whole world under the sky; the fiery and hand made pillars of the holy catholic church, from one end to the other; the intellectual, lofty and luminous clouds of the spiritual storms and gifts of the divine; the holy stones rolling and coming down from the highest mountains, which stand above the heavens and which crush and shatter every siege engine and tower ${ }^{15}$ of evil on earth; the gold-gleaming eagles with great wings, hunting after all animals, both savage and intelligent, all over the fields. About them we spoke as one should.

9. Multiple and great are the concepts of your archangelic power and dignity, because of which we were induced to praise your magnificence, but weak and small is the faculty of our tongue. Broad, really aloft and manifold is the picture of your incorporeal, immaterial and all-comely form, but petty, base and unskillful the account coming forth through the bodily organs from our heart. How, then, would we duly praise you, captains of the almighty God? What incorporeal appropriate speech would we bring forth to you, commanders of the disembodied and spiritual, with our material and bodily lips? What sounds would we use to denote the unutterable and unspeakable glory of yours? Any earthborn creature really fails in lauding you, heavenly creatures, properly. For things that our eye has not seen, ${ }^{16}$ nor our ear heard, nor even the intellectual thoughts of our heart imagined, ${ }^{17}$ of these good things you always have the glory and blessed enjoyment. Thus, hidden and secret from anyone is your glory, unutterable your life, undepictable the nature of your magnificence. Surely then, we thought fit ${ }^{18}$ to honor

14 The combination of $L c .15,7$ and Eph. 5, 1 in the same context is also found in Metrophanes, Homilia 2, 22 (p. 118, 1. 32-35).

15 This combination of rather rare words can also be found in Metrophanes' In Ecclesiasten 7, 1, 43 and 9, 3, 13-14.

16 For the use of " $\delta \varepsilon v$ in Metrophanes, see EtTLinger/NoRet, Commentarius (as footnote 4 above) $\mathrm{xl}$.

17 The same quote can be found in the first lines of the Vita Polycarpi of Metrophanes (BHG

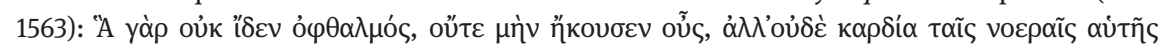

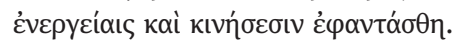

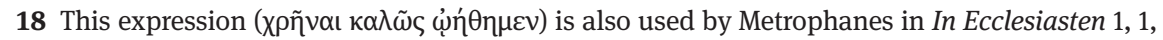
24-25 and 1, 4, 124. 
you silently, rather than with words, as the indescribable attendants and envoys of the unattainable and secret glory and of the beauty and splendor, worthy of God. For, whenever the mind is weakened and slackens in relation to the faculty of imagination and contemplation of the supra-natural, the spoken word would not suffice to be brought into line with the sublimity of what has been imagined, nor is able to produce the expression of what has been apprehended in whatever way, and in this case, silence rather is to be chosen above and is more profitable than speech, ${ }^{19}$ as it is better for those who in no way have acquired wings to stay at their suitable and circumscribing place than to fly like birds to the highest sky or to cross and go across a vast sea.

10. Let us, then, yet for the remaining keep silence, since we no longer dare to discuss the mighty works of the eulogies of yours in detail. We veil ourselves in speechlessness rather than to try to relate circumstantially your sublimity that is beyond understanding and to rush through the accounts of your greatest splendor. But have pity, also now as always, upon the human earthly-mindedness and way of life, you sublime first citizens of the kingdom of heaven. Move earnest entreaties to the common Lord and Creator to pardon us, who are subject to weakness and hardship of wily mistakes. Persuade with your archangelic arguments the good, just God, who, by his goodness, is the only one not to show partiality, to justify us rather than to condemn us to the yoke of justice; to send down mercy to us through you, the good and men loving angels, but not to send out anger and affliction, viz. a bestowal by means of evil angels; to be patient with our mistakes and sins; to lead all of us, who have placed our hope in Him, to improvement through repentance and to change towards the better; to save us, but not to condemn us together with the universe by you passing over us in silence; moreover, to make to rise for us, along with the rays of the perceptible sun, also way more the radiant beams of the specific, most useful and benevolent providence; to enligthen the intellectual eyes of our hearts to make us understand and apprehend the greatness of His wonders and to make us take the roads of divine justice and lead a saint like life; but in no way to keep back and hide from us the ligths and torches of His inscrutable mercy and divine forethought.

11. Verily, archangelic pair of the divine Trinity, men loving yoke of the men loving, monarchial Lord, always offer those, who are of the same faith and mind as you, your interceding help, and procure for us the divine favor and assistance from above..$^{20}$ Deliver us all from evil and rage of crooked Satan, who fights

19 Such exhortation towards silence rather than to speech is also expressed by Metrophanes in In Ecclesiasten 1, 11, 20 - 30.

20 The same expression can be found in Metrophanes' In Ecclesiasten 9, 19, 28 -29 and 9, 22, 30. 
against God and hates men. ${ }^{21}$ Make, with your constant entreaties to the King of glory, of no effect his devices and wiles, by which he contrives to draw all the inhabitants of the earth towards meanness and wickedness. Lower the raised eyebrow, and make completely clear with your appeals that boasting is idle and vain. Pluck out the tares seeded by the enemy in the field of the church, and declare that the seed of the Master and the Lord is pure. Beseech the divine Author and King of peace to mightily give to all Christians peace and harmony with Him, so that His glorified and magnificent name would, just as in heaven, be glorified by all also on earth with proper consent and necessary agreement. Prepare the phalanges of the unbelieving nations ${ }^{22}$ to be defeated and to be put to flight. Make, as commanders-in-chief of the God of three suns and the King of glory, war together with the armies and commanders, more precisely with the Christian kings of our inhabited world and society. Put to shape the wicked, the infidel, fill their faces with dishonor, so that they too would get to know our harmony with you and would understand that you are the helpers and allies of those who are of the same faith and glory. Show ${ }^{23}$ clearly as commanders-in-chief of the Christian churches as well as of their armies, you commanders-in-chief of the powers of above and champions of the assembly of the firstborn who are enrolled in heaven. Strengthen us with your interceding help, and strengthen forever our power to do without interference the will of the common Lord and God of all, just like you. Lead us too to the path of salvation by your favor. ${ }^{24}$ Guide us, unworthy and humbles ones, to your spiritual and unsubstantial communion. Deliver us from degrading passions. Free us from every misdeed and malice. ${ }^{25}$ Take your place in our circle, and chase away those who fight against us from the height of their own evil as our spiritual enemies. Reform the choices of men acting wickedly. Silence the counsels of those hateful to us. Make us stronger than life's temptations and every source of sin. Cover and guard our lives with your archangelic wings, and redeem us from every damage.

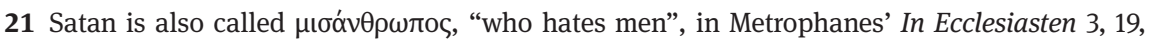
$36-37$.

22 According to Georgiadès, Metrophanes alludes to the Russians.

23 For the correction of the manuscript reading $\delta \delta \delta \alpha ́ \xi \alpha \tau \varepsilon$ into $\delta \varepsilon i ́ \xi \alpha \tau \varepsilon$, proposed by Georgiadès and

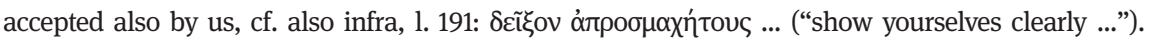

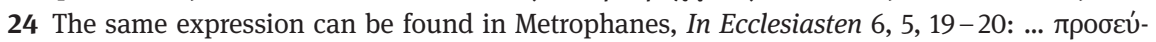

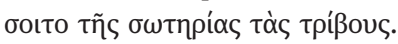

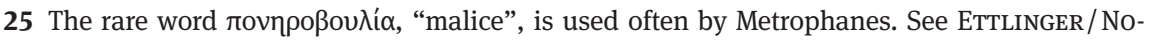
RET, Commentarius (as footnote 4 above) li; VAN Deun, Métrophane (as footnote 1 above) 359. Moreover, the combination with каколрауí $\alpha$ can also be found in Metrophanes' In Ecclesiasten $3,23,63-64$. 
12. Verily, Michael, whose name is explained by "general of God", ${ }^{26}$ always wage war in behalf of us, and deliver us from every harm by our spiritual and physical adversaries. Verily, Gabriel, whose name is explained by "courage of God", ${ }^{27}$ gird on to us the courage of your interceding help, and prove us to be immune to the contrivances of evil. ${ }^{28}$

13. Michael and Gabriel, blessed pair of the unitary divinity of three persons, be the champions and helpers of our hardship, so that we would be deemed worthy to live on earth in peace and perfect justice, just as we, along with you, hold citizenship in heaven and set our minds on things that are above, and so that we, at the end of our lives, would acquire the renowned fruits of virtue's good labors, and would be translated from this imitative life, subject to many passions, to the true, by your favor uncompounded, blessed and divine life, ${ }^{29}$ and that also we, along with you, would glorify incessantly the King of glory, ${ }^{30}$ Christ and our God. Because to Him is all the glory, honor and obeisance, together with the almighty Father and all-holy, good and life giving Spirit, now and forever and to the infinite ages of ages. Amen. ${ }^{31}$

26 For this etymology, see F. WuTz, Onomastica sacra. Untersuchungen zum Liber interpretationis nominum Hebraicorum des hl. Hieronymus. TU 41.1-2. Leipzig 1915, 184-186 and 701. See also T. MatantSEva, Éloge des archanges Michel et Gabriel par Michel le moine (BHG 1294a). JÖB 46 (1996) 121; 121, note 91 (BHG 1294a, which also contains this etymology of Michael [7, 28 and 10,6], seems to date to the same period as our text). It is noteworthy that Metrophanes really likes to give etymological explanations of biblical names; see, e. g., Hostens, Dissertatio (as footnote 4 above) xxxiv-xxxv; Metrophanes, In Ecclesiasten 1, 2, 68 and 109-110.

27 For this etymology of Gabriel, see WuTz, Onomastica sacra (as footnote 26 above) ibidem.

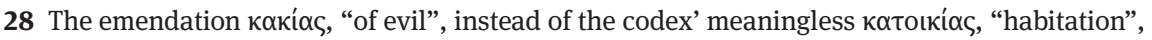
was suggested first by Georgiadès and is, according to us, very reasonable, as $\mu \eta \chi \alpha v \eta \dot{\mu \alpha} \tau \alpha$ iñ $\varsigma$

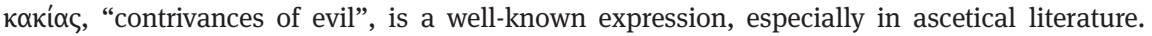

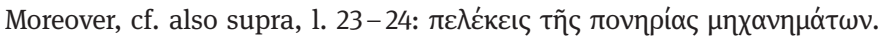

29 The same idea (even with some words repeated) can also be found in Metrophanes' In Ecclesiasten 3, 24, 39-44.

30 At the very end of Homilia 4, 54 (p. 173, 1. 29-30), just before the doxology as well, the same

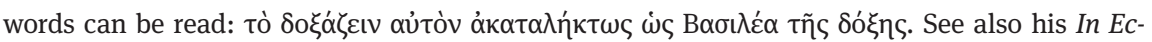

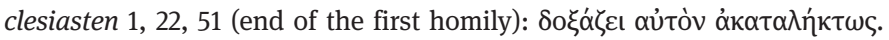

31 The very same doxology can also be found in Metrophanes, Homilia 4, 54 (p. 173, 1. 30 -33). 


\section{Index Biblicus}

Gen. 3, 161.41

Gen. 3, 24 1. 22

Tob. 5, 221.1

III Mach. 2, 2 1. 27

Iob 7, 1 1. 185

Ps. 2, 5 1. 177

Ps. 16, 8 1. 186-187

Ps. 23, 7-10 1. 4; 78; 156-157; 170; 200

Ps. 24, 5 1. $178-179$

Ps. 30,18 1. $170-171$

Ps. 32, 10 l. 185

Ps. 55, 3 1. 182

Ps. 60, 5 1. $186-187$

Ps. 71, 8 1. 99-100

Ps. $82,171.171$

Ps. $119,41.24$

Od. 7, $391.93-94$

Sap. 3, 15 1. 197-198

Sap. 7, 26 1. 19

Zach. 9, 16 1. 101-102

Mal. 3, 201.78

Is. 45, 2 1. 102-103

Is. 64,4 1. 116-117

Ier. 27, 291.182

Ez. 17, 3 1. 104

Ez. 17, 7 1. 104

Dan. (Theodot.) 3, $391.93-94$

Dan. (Theodot.) 8, 15-26 1. 33-35

Dan. (Theodot.) 9, 21-25 1. 44-52

Dan. (Theodot.) 10, 5-6 1. 59-62

Dan. (Theodot.) 10, $12-14$ 1. 62-69

Dan. (Theodot.) 10, $211.69-70$
Mt. 6, 9 1. 88

Mt. 13, 25 1. 161

Mt. 18, 10 1. 86-88

Lc. 1, 11-20 l. 33-35

Lc. 1, 26-28 1. 39-40

Lc. 1, 26-38 1. 33-35

LC. 15, 7 1. 88; 90

LC. $15,9-101.88$

Lc. $15,101.90$

Io. 3, 17 1. 145

Io. 17,3 1. 7 - 8

Act. 7, 38 1. 32-33

Act. 7, $531.32-33$

Act. 24, 15 1. 58

Rom. 1, 26 1. 181

Rom. 3, 111.93

Rom. 9, 5 1. 79

Rom. 11, 33 1. 151

I Cor. 2, 9 1. 116-117

II Cor. 3, 10 1. 165-166

Eph. 1, 18 1. 147-148

Eph. 5, 11.91

Eph. 6, 11 1. 22-23; 157

Phil. 3, 20 1. 6; 196

Col. 3, 2 1. 196

Hebr. 4, 121.118

Hebr. 5, 2 1. 137

Hebr. 7, 2 1. 163

Hebr. 12, 23 1. 175-176

Iac. 1, 17 1. 27-28

Ep. Iud. 9 1. $81-84$ 
Brought to you by | KU Leuven University Library Authenticated | erika.gielen@hiw.kuleuven.be author's copy Download Date | 2/5/16 10:17 AM 